\title{
EXTRACCIÓN Y CARACTERIZACIÓN DEL ALMIDÓN DE TRES VARIEDADES DE QUINUA (Chenopodium quinoa Willd) NEGRA COLLANA, PASANKALLA ROJA Y BLANCA JUNÍN
}

\author{
Doyla Arzapalo Quinto ${ }^{a}$, Katty Huamán Cóndora, Miguel Quispe Solano*a, \\ Clara Espinoza Silva ${ }^{b}$
}

\begin{abstract}
RESUMEN
Se obtuvo y caracterizó química, fisicoquímica y funcionalmente, el almidón de tres variedades de quinua: negra Collana (VC), Pasankalla roja (VP) y blanca Junín (VB). Los análisis químicos proximales para grano y almidón de quinua se realizaron por el método AOAC (2000); la extracción de almidón se realizó a escala de laboratorio; el mayor rendimiento es $30,62 \%$ en la VB, seguida por VP con $26,71 \%$; mientras VC obtuvo el mínimo $18,95 \%$, existe diferencia significativa en $(\mathrm{p}<0,05)$ entre las variedades. Para las propiedades funcionales, las muestras de almidón presentan una baja solubilidad de $5 \%$ y restringido poder de hinchamiento de $0,7 \%$, por lo que no existen diferencias significativas en $(p>0,05)$, comprobando que sus gránulos tienen fuerzas o enlaces de unión muy fuertes, se mostró un alto rango de temperatura de gelatinización $\left(66-69^{\circ} \mathrm{C}\right)$ donde no hay diferencia significativa ( $\mathrm{p}>0,05)$; además, se observó un ligero pico de viscosidad de $2006 \mathrm{cp}$ para $\mathrm{VC}$, $1521 \mathrm{VB}$ y un mínimo de $1009 \mathrm{cp}$ en VP. Teniendo como resultado una extrema estabilidad al descongelamiento de 4,9VC; 1,3VP; 1,3VB y una baja retrogradación (41,4VC; 70,7VP y $72,3 \mathrm{VB})$; para ambos se encontró diferencias significativas en $(\mathrm{p}<0,05)$, sugiriendo su uso en productos sometidos a estos procesos.
\end{abstract}

Palabras clave: Almidón, quinua, caracterización, propiedades funcionales, retrogradación.

\section{EXTRACTION AND CHARACTERIZED STARCH THREE VARIETIES OF QUINOA (Chenopodium quinoa Willd) BLACK COLLANA, RED PASANKALLA AND WHITE JUNÍN}

\begin{abstract}
We have obtained and characterized chemical, physicochemical and functional starch quinoa three varieties: black Collana (VC), red pasankalla (VP) and white Junín (VB). Proximal chemical analysis and grain quinoa starch were performed by the AOAC method (2000), starch extraction was performed in laboratory scale; the highest yield is $30.62 \%$ in the VB followed by VP with $26.71 \%$, while VC won at least $18,95 \%$; there is significant difference at $(p<0.05)$ between varieties. For functional properties of starch samples have low solubility of $5 \%$ and restricted swelling power of $0.7 \%$ so that there are no significant differences ( $\mathrm{p}>$ $0.05)$ granules are finding that their forces or links very strong union; a high gelatinization temperature range $\left(66-69^{\circ} \mathrm{C}\right)$ which showed no significant difference $(\mathrm{p}>0.05)$; plus a slight peak viscosity of 2006cp to VC, $1521 \mathrm{VB}$ and minimum VP $1009 \mathrm{cp}$. Resulting in extreme

a Facultad de Ciencias Aplicadas, Escuela Académico Profesional de Ingeniería Agroindustrial, Universidad Nacional del Centro del Perú, quispe_miguelangel@hotmail.com

b Facultad de Ingeniería en Industrias Alimentarias, Universidad Nacional del Centro del Perú, cespinoza@hotmail.com
\end{abstract}


stability $4.9 \mathrm{VC}$ thawing; $1.3 \mathrm{VP} ; 1.3 \mathrm{VB}$ and low retrogradation $(41.4 \mathrm{VC} ; 70.7 \mathrm{VP}$ and $72.3 \mathrm{VB}$ ); to two significant differences were found $(\mathrm{p}<0.05)$, suggesting its use in products subject to these processes.

Key words: Starch, quinoa, characterization, functional properties, retrogradation.

\section{INTRODUCCIÓN}

El polisacárido más utilizado en la industria alimentaria como ingrediente esencial, por su gran versatilidad y su costo relativamente bajo, es el almidón. Debido a sus propiedades fisicoquímicas y funcionales los almidones se emplean como: agentes espesantes para incrementar la viscosidad de salsas y potajes, como agentes estabilizantes de geles o emulsificantes, como elementos ligantes y agentes de relleno ${ }^{1}$.

La extracción y caracterización del almidón nativo proveniente de diferentes fuentes no convencionales, como la quinua, puede proveer materias primas con propiedades especiales para usos específicos en la industria.

La quinua, es un grano cultivado en el Perú desde épocas prehispánicas; este cultivo ha sido considerado por la FAO como uno de los alimentos del futuro a nivel mundial por su gran capacidad de adaptación agronómica. Existen alrededor de 100 cultivares de quinua, cuyos granos son preparados de diversas maneras para su consumo directo y transformados en múltiples derivados; entre ellos, las variedades: Collana negra, Pasankalla roja y blanca Junín ${ }^{2}$.

Los carbohidratos de la semilla de la quinua contienen entre 58 a $68 \%$ de almidón; se encuentran localizados en el perisperma en gránulos pequeños $(2 \mu \mathrm{m})$, siendo más pequeños que los granos comunes; son parcialmente cristalinos e insolubles en agua a temperatura ambiente; los tamaños y formas dependen de la fuente biológica; es altamente digerible ${ }^{3}$.

El almidón de la quinua ha sido estudiado muy poco. Sería importante estudiar sus propiedades funcionales. El almidón de quinua tiene una excelente estabilidad frente al congelamiento y la retrogradación ${ }^{4}$. Estos almidones podrían ofrecer una alternativa interesante para sustituir almidones modificados químicamente ${ }^{5}$. La variación genética del tamaño de gránulo de almidón de la colección boliviana de quinua fluctuó entre 1 a $28 \mu \mathrm{m}$, permitiendo esta variable dar una orientación agroindustrial ${ }^{6}$.

\section{PARTE EXPERIMENTAL}

La presente investigación es de tipo aplicada porque se utilizó métodos, técnicas y procedimientos, para solucionar problemas y producir resultados de utilidad práctica ya estudiados.

Tiene alcance de nivel descriptivo-explicativo porque se describe el fenómeno, su situación y su contexto; además, especifica las propiedades y características del objeto de estudio.

\section{Materiales y reactivos}

Se trabajó con tres variedades de quinua (Collana negra, Pasankalla roja y blanca Junín) provenientes de las principales zonas de producción de quinua (Ayacucho, Puno y Junín). Se utilizó $9 \mathrm{~kg}$ de almidón de quinua; siendo $3 \mathrm{~kg}$ de muestra de cada variedad.

\section{Etapa 1: Obtención de las muestras o unidades experimentales}

Se realizó mediante una técnica de muestreo, los proveedores fueron ubicados de las principales zonas de producción de quinua a nivel nacional (Ayacucho, Puno y Junín). 


\section{Etapa 2: Evaluación de la materia prima y extracción del almidón}

Se realizó el análisis químico proximal y fisicoquímico para materia prima, y se obtuvo el rendimiento de almidón. Se determinó (humedad y materia seca, proteína, grasa, fibra, ceniza, carbohidratos, $\mathrm{pH}$, acidez y rendimiento de almidón).

El flujograma de proceso de operaciones para la obtención de almidón se detalla en la figura 1.

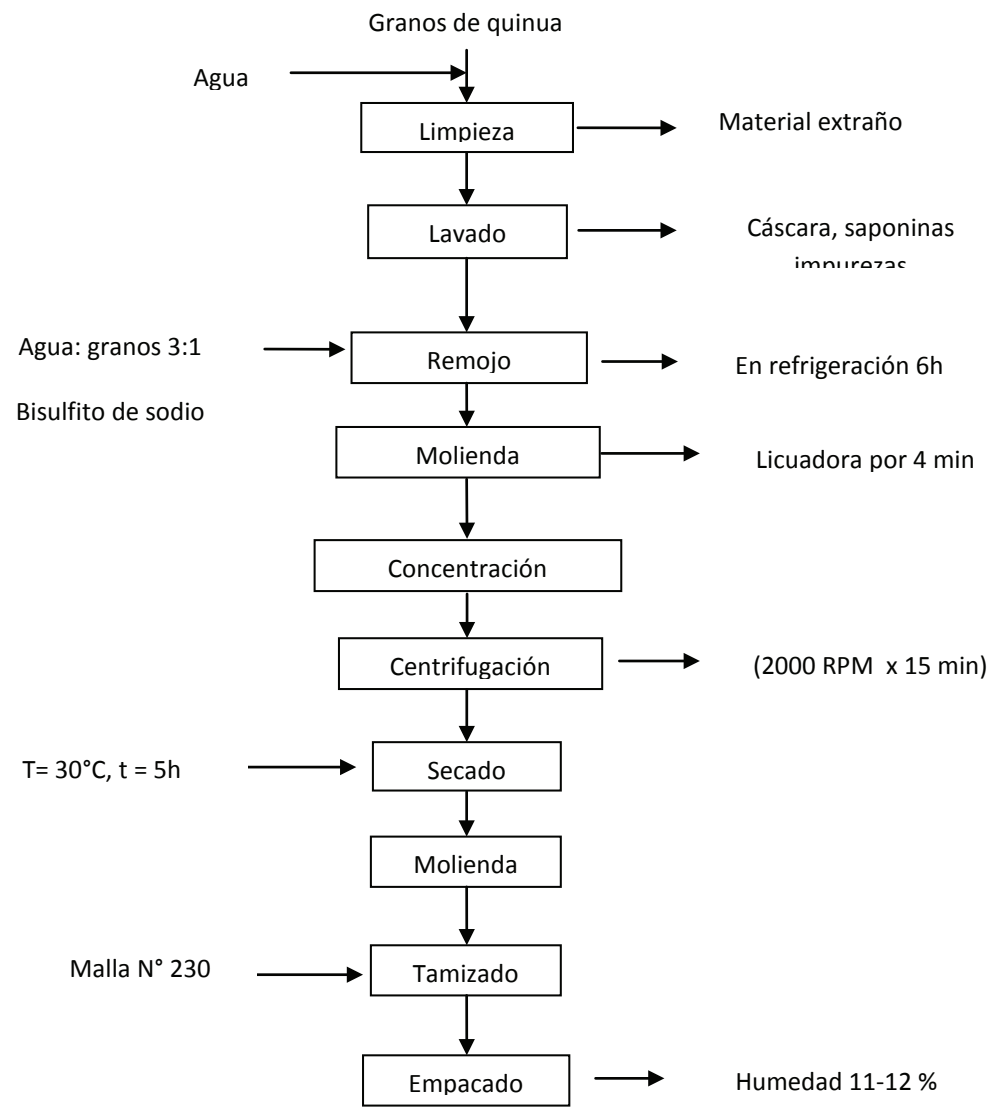

Figura 1. Flujograma de obtención de almidón de quinua.

\section{Etapa 3: Evaluación de la calidad del almidón}

Se evaluó las características químicas, fisicoquímicas, propiedades físicas y funcionales de las tres variedades de almidón.

Análisis químico-proximal y fisicoquímico. Se determinó (humedad y materia seca, proteína, grasa, fibra, ceniza, carbohidratos, $\mathrm{pH}$, acidez y rendimiento de almidón).

Análisis de propiedades físicas

Índice de finura - granulometría ${ }^{7}$

Colorimetría - Determinación del color en el sistema CIELAB ${ }^{7}$ 


\section{Análisis de propiedades funcionales}

Viscosidad $^{7}$

Temperatura de gelatinización ${ }^{8}$

Índice de solubilidad de agua ${ }^{9}$

Índice de absorción de agua ${ }^{9}$

Poder de hinchamiento ${ }^{9}$

Retrogradación ${ }^{10}$

Estabilidad al descongelamiento ${ }^{11}$

\section{Parámetros de calidad de almidón}

Anhídrido sulfuroso ${ }^{12}$

Material no amiláceo ${ }^{13}$

\section{Análisis estadístico}

Para la evaluación estadística se utilizó un DCA; el análisis de varianza se trabajó con 0,05 de significancia; al encontrar diferencia significativa se procedió a realizar la prueba de comparaciones de medias Tukey a un nivel de $\alpha=0,05$. Los datos fueron procesados con la ayuda de los programas estadísticos SPSS v_19, y la hoja de cálculo Microsoft Excel 2010.

\section{RESULTADOS Y DISCUSIÓN}

\section{Caracterización químico proximal y fisicoquímico de las tres variedades de quinua. Químico proximal}

Se desarrolló, según lo detallado en la metodología mediante tablas simples; los resultados se reportan en la tabla 1.

Tabla 1. Composición químico proximal de las tres variedades de quinua.

\begin{tabular}{|c|c|c|c|c|c|c|}
\hline \multirow[b]{2}{*}{ COMPONENTES } & \multicolumn{2}{|l|}{$\mathrm{VC}$} & \multicolumn{2}{|l|}{ VP } & \multicolumn{2}{|l|}{ VB } \\
\hline & Base húmeda & $\begin{array}{l}\text { Base } \\
\text { seca }\end{array}$ & Base húmeda & $\begin{array}{l}\text { Base } \\
\text { seca }\end{array}$ & Base húmeda & $\begin{array}{l}\text { Base } \\
\text { seca }\end{array}$ \\
\hline Humedad & $11,50 \pm 0,45 \mathrm{~ns}$ & --------- & $11,66 \pm 0,04 \mathrm{~ns}$ & --------- & $11,34 \pm 0,01 \mathrm{~ns}$ & ---------- \\
\hline Proteína & $9,90 \pm 0,00 \mathrm{c}$ & 11,19 & $11,21 \pm 0,00 \mathrm{~b}$ & 12,68 & $12,11 \pm 0,00 \mathrm{a}$ & 13,64 \\
\hline Lípidos & $4,67 \pm 0,00 \mathrm{c}$ & 5,31 & $5,21 \pm 0,00 \mathrm{~b}$ & 5,89 & $6,32 \pm 0,00 \mathrm{a}$ & 7,10 \\
\hline Fibra & $3,21 \pm 0,02 \mathrm{c}$ & 3,6 & $3,52 \pm 0,00 \mathrm{~b}$ & 3,96 & $4,28 \pm 0,00 \mathrm{a}$ & 4,84 \\
\hline Ceniza & $2,62 \pm 0,01 \mathrm{c}$ & 2,94 & $3,12 \pm 0,47 b$ & 3,5 & $3,86 \pm 0,09 \mathrm{a}$ & 4,40 \\
\hline Carbohidratos & $71,30 \pm 0,46 \mathrm{a}$ & 80,56 & $68,79 \pm 0,45 b$ & 77,92 & $66,37 \pm 0,11 \mathrm{c}$ & 74,85 \\
\hline Energía & 367,12 & ------ & 366,84 & -------- & 370,71 & -------- \\
\hline
\end{tabular}


En la tabla 1 se reportó los resultados del análisis químico proximal de las tres variedades de quinua; en cuanto al porcentaje de humedad se puede apreciar que no existe diferencia con ( $\mathrm{p}>0,05$ ); al respecto, $\mathrm{MINAG}^{2}$ indica que el porcentaje de humedad para la quinua debe ser como un máximo $12 \%$, por lo tanto, las tres variedades se encuentran dentro del parámetro establecido. Para los carbohidratos, el mayor porcentaje lo obtuvo la variedad negra Collana con un 71,30\%, seguido de la variedad roja Pasankalla con 68,79\% y la de menor porcentaje 66,37\% la variedad blanca Junín. Estos valores son mayores a los reportados por otros autores, haciendo de esta una mejor opción para la obtención de almidón. La quinua contiene de 58,1-64,2 \% de almidón, encontrándose en forma de gránulos ${ }^{16}$. Para Ahamed et al. ${ }^{4}$ el almidón de quinua de las variedades, rojo, amarillo y blanco, es de 59, 58 y $64 \%$, respectivamente. Con respecto a la proteína existe diferencia significativa con $(p<0,05)$. Al realizar una comparación con la papa y el camote, que son fuentes importantes de almidón, los valores son ( $2 \%$ y $1,4 \%)$, respectivamente; por lo tanto, este componente es más alto en la quinua, por lo que la extracción del almidón debería ser más exhaustiva con el fin de reducir al mínimo estos componentes, ya que pueden interferir en las propiedades del almidón.

\section{Análisis fisicoquímico}

Tabla 2. Análisis de $\mathrm{pH}$ y acidez de las tres variedades de quinua.

\begin{tabular}{lccc}
\hline & VC & VP & VB \\
\hline $\begin{array}{l}\text { Acidez (meq de ácido } \\
\text { sulfúrico } / \mathrm{g})\end{array}$ & $0,12 \pm 0,00 \mathrm{a}$ & $0,12 \pm 0,00 \mathrm{c}$ & $0,14 \pm 0,00 \mathrm{~b}$ \\
$\mathrm{pH}\left(20^{\circ} \mathrm{C}\right)$ & $6,45 \pm 0,07 \mathrm{~ns}$ & $6,20 \pm 0,14 \mathrm{~ns}$ & $6,70 \pm 0,14 \mathrm{~ns}$ \\
\hline *, ns: Indican significancia a $p<0,05$ y no significante. Valores con diferentes letras dentro de \\
cada columna denotan significancia en la prueba de Tukey $(p<0,05)$, valores promedio de 2 \\
repeticiones \pm desviación estándar.
\end{tabular}

La tabla 2 muestra los resultados del análisis de acidez para las tres variedades de quinua; están expresados en meq. de ácido sulfúrico /g; estas se encuentran dentro de los rangos establecidos para almidones, en general. Con respecto al análisis de $\mathrm{pH}$ de las tres variedades de quinua los valores fueron $(6,70 ; 6,20$ y 6,45). Se realizó el ANOVA cuyo resultado nos da a conocer que no existe diferencia significativa entre las muestras $(p>0,05)$.

\section{Rendimiento del almidón}

El rendimiento del almidón, es la fracción (\%) que resulta después de cada operación unitaria; iniciándose con la recepción de la materia prima al 100\%; los resultados se reportan en la tabla 3 .

Tabla 3. Rendimiento del almidón de tres variedades de quinua.

\begin{tabular}{cccc}
\hline VC & VP & VB * \\
\hline Rendimiento (\%) & $18,95 \pm 0,05 \mathrm{c}$ & $26,71 \pm 0,15 \mathrm{~b}$ & $30,62 \pm 0,03 \mathrm{a}$ \\
\hline *, ns: Indican significancia a $p<0,05$ y no significante. Valores con diferentes \\
letras dentro de cada columna denotan significancia en la prueba de Tukey \\
$(p<0,05)$, valores promedio de 2 repeticiones \pm desviación estándar.
\end{tabular}


De la tabla 3 se puede observar que hay diferencia significativa con $(p<0,05)$ entre el porcentaje de rendimiento de almidón de las tres variedades. Matos y Sánchez3 determinaron el rendimiento del almidón de tres variedades de quinua, Pasankalla, Salcedo INIA y Kancolla, el mayor rendimiento es de 47,39 \% para Salcedo INIA; seguido de la variedad Kancolla, de $38,46 \%$; mientras que el menor rendimiento es de $18,25 \%$ para la variedad Pasankalla. Cabe destacar que estos valores superan al porcentaje de fuentes importantes de almidón como la papa, camote, yuca y maíz (7-18\%). Esta diferencia, comparada con los resultados obtenidos, se debe a las variedades en estudio y a las condiciones climatológicas en las que se desarrollan. Ello está corroborado por Singh et al. ${ }^{14}$ donde la relación amilosa/amilopectina en los almidones varía de acuerdo al origen botánico, al clima y tipo de suelo, al proceso de obtención y purificación, así como de las condiciones de almacenamiento.

\section{Caracterización de la composición químico proximal y fisicoquímico del almidón. Análisis químico proximal}

Tabla 4. Análisis químico proximal del almidón obtenido de las variedades quinua.

\begin{tabular}{|c|c|c|c|c|c|c|}
\hline \multirow[b]{2}{*}{ COMPONENTES } & \multicolumn{2}{|l|}{$\mathrm{VC}$} & \multicolumn{2}{|l|}{$\mathbf{V P}$} & \multicolumn{2}{|l|}{ VB } \\
\hline & Base húmeda & $\begin{array}{l}\text { Base } \\
\text { seca }\end{array}$ & Base húmeda & $\begin{array}{l}\text { Base } \\
\text { seca }\end{array}$ & Base húmeda & $\begin{array}{l}\text { Base } \\
\text { seca }\end{array}$ \\
\hline Humedad & $11,12 \pm 0,29 \mathrm{~ns}$ & ------ & $11,22 \pm 0,94 \mathrm{~ns}$ & ------ & $11,10 \pm 0,04 \mathrm{~ns}$ & ----- \\
\hline Proteína & $0,20 \pm 0,00 \mathrm{c}$ & 0,23 & $0,24 \pm 0,00 \mathrm{~b}$ & 0,27 & $0,28 \pm 0,00 \mathrm{a}$ & 0,31 \\
\hline Lípidos & $3,71 \pm 0,00 \mathrm{c}$ & 4,18 & $4,22 \pm 0,00 \mathrm{~b}$ & 4,78 & $5,01 \pm 0,00 \mathrm{a}$ & 5,66 \\
\hline Fibra & $1,74 \pm 0,02 \mathrm{c}$ & 1,96 & $1,80 \pm 0,03 \mathrm{~b}$ & 2,04 & $2,77 \pm 0,03 \mathrm{a}$ & 3,13 \\
\hline Ceniza & $0,27 \pm 0,01 \mathrm{c}$ & 0,30 & $0,41 \pm 0,02 \mathrm{a}$ & 0,46 & $0,35 \pm 0,00 \mathrm{~b}$ & 0,40 \\
\hline Carbohidratos & $80,98 \pm 0,27 \mathrm{a}$ & 93,53 & $75,19 \pm 0,93 \mathrm{~b}$ & 93,00 & $73,78 \pm 0,04 \mathrm{c}$ & 90,96 \\
\hline Energía & 366,03 & ------- & 367,42 & ------- & 368,19 & ----- \\
\hline
\end{tabular}

*, ns: Indican significancia a $p<0,05$ y no significante. Valores con diferentes letras dentro de cada columna denotan significancia en la prueba de Tukey $(p<0,05)$, valores promedio de 2 repeticiones \pm desviación estándar.

Nota: los datos obtenidos se realizaron en función en normas establecidas por AOAC (2000)

De la tabla 4 se puede observar que no hay diferencia significativa con $(p>0,05)$ entre el porcentaje de humedad de las tres variedades de almidón de quinua. El contenido de humedad en los gránulos de almidón va de un 10 al 12\% en cereales y de 14 a $19 \%$ en almidones de tubérculos y raíces. Para lípidos hay diferencia significativa con $(\mathrm{p}<0,05)$. Guizar $^{15}$ menciona que los lípidos en almidones de tubérculos, como la papa, el camote y la tapioca es muy bajo $(<0,1 \%)$ comparado con los almidones provenientes de cereales (maíz, trigo, arroz, cebada y sorgo), los cuales contienen de 0,6 a 1,0\% de lípidos; nuestros resultados para quinua son superiores. Para la fibra, Raygada ${ }^{16}$, en su investigación, determinó valores inferiores comparados a los nuestros, estos son 0,10 y $0,11 \%$ para almidón de quinua Chullpi y Kancolla; concluye que aún estos son valores muy altos; otros autores afirman también que el almidón de quinua no debería presentar fibra. 
Con respecto a la proteína, existe diferencia significativa con $(\mathrm{p}<0,05)$. Raygada ${ }^{16}$, en su investigación, reporta que el almidón de quinua de las variedades Kancolla y Chullpi contiene $(0,45$ y $0,30 \%)$ de proteína total, respectivamente. En nuestra investigación la proteína está en un rango de $0,20-0,28 \%$.

\section{Análisis fisicoquímico}

Tabla 5. Análisis de pH y acidez de almidón de las tres variedades de quinua.

\begin{tabular}{lcccc}
\hline & VC & VP & VB \\
\hline Acidez (meq de ácido & $0,03 \pm 0,00 \mathrm{~b}$ & $0,03 \pm 0,00 \mathrm{a}$ & $0,02 \pm 0,00 \mathrm{c}$ \\
sulfúrico $/ \mathrm{g})$ & $6,07 \pm 0,17 \mathrm{~ns}$ & $6,20 \pm 0,05 \mathrm{~ns}$ & $6,49 \pm 0,05 \mathrm{~ns}$ \\
$\mathrm{pH}\left(20^{\circ} \mathrm{C}\right)$ & & 6,050
\end{tabular}

*, ns: Indican significancia a $p<0,05$ y no significante. Valores con diferentes letras dentro de cada columna denotan significancia en la prueba de Tukey $(p<0,05)$, valores promedio de 2 repeticiones \pm desviación estándar.

En la tabla 5 se reporta los valores de $\mathrm{pH}$; no existe diferencia significativa con $(\mathrm{p}>0,05)$. Según ISI17 el $\mathrm{pH}$ a $20^{\circ} \mathrm{C}$ en el almidón nativo debe estar entre $6,0-6,5$; las muestras analizadas están dentro de este rango. Los valores de la evaluación de acidez nos permite interpretar que no existe diferencia significativa. Según ISI $^{17}$, la acidez titulable debe estar 0,02 y 0,05 meq de ácido sulfúrico/g en caso de almidones.

Caracterización de las propiedades físicas y funcionales del almidón obtenido de las tres variedades de quinua, mediante evaluaciones estadísticas.

Propiedades físicas

Tabla 6. Evaluación de índice de finura y colorimetría del almidón de quinua.

\begin{tabular}{cccc}
\hline & $\mathbf{V C}$ & $\mathbf{V P}$ & $\mathbf{V B}$ \\
\hline Índice de finura (\%) & $1,35 \pm 0,05 \mathrm{~ns}$ & $1,25 \pm 0,02 \mathrm{~ns}$ & $1,11 \pm 0,11 \mathrm{~ns}$ \\
\hline Colorimetría $\left(\mathbf{L}^{*}\right)$ & $42,29 \pm 2,72 \mathrm{c}$ & $66,11 \pm 4,21 \mathrm{~b}$ & $80,89 \pm 1,30 \mathrm{a}$ \\
\hline
\end{tabular}

$*$, ns: Indican significancia a $p<0,05$ y no significante. Valores con diferentes letras dentro de cada columna denotan significancia en la prueba de Tukey $(p<0,05)$, valores promedio de 2 repeticiones \pm desviación estándar.

Según la tabla 6 , no hay diferencia significativa con $(\mathrm{p}>0,05)$ entre el porcentaje de índice de finura de almidón de las tres variedades de quinua. En nuestro estudio se utilizó la malla número 100; por lo tanto, están dentro de los parámetros aceptables para almidones ${ }^{7}$.

Los resultados de la evaluación del color en las tres variedades de quinua nos indican que existe diferencia significativa con $(\mathrm{p}<0,05)$. ICONTEC ${ }^{17}$ menciona que la medida del color servirá como una medida de la calidad homogénea, en tanto que $\mathrm{L}^{*}$ indica la luminosidad de la muestra; esta va de 0 (negro) a 100 (blanco) lo que generalmente se utiliza para evaluar el color del almidón; el centro del diagrama es acromático; esto quiere decir que; conforme 
se incrementan los valores, va aumentando la saturación del color, encontrando así que el almidón que presenta mejores características de calidad en cuanto al color es procedente de la variedad blanca Junín, seguido por Pasankalla roja y por último Collana negra.

\section{Propiedades funcionales}

Tabla 7. Evaluación de las propiedades funcionales del almidón.

\begin{tabular}{|c|c|c|c|}
\hline $\begin{array}{l}\text { PROPIEDADES } \\
\text { FUNCIONALES }\end{array}$ & VC & VP & VB \\
\hline Viscosidad (cp) & $2006,50 \pm 2,12 \mathrm{a}$ & $1009,00 \pm 1,41 b$ & $1521,50 \pm 9,19 \mathrm{c}$ \\
\hline Gelatinización $\left({ }^{\circ} \mathrm{C}\right)$ & $65,50 \pm 0,71 \mathrm{~ns}$ & $68,00 \pm 1,41 \mathrm{~ns}$ & $68,50 \pm 0,71 \mathrm{~ns}$ \\
\hline Solubilidad (\%) & $5,03 \pm 0,01 \mathrm{~ns}$ & $4,94 \pm 0,12 \mathrm{~ns}$ & $4,97 \pm 0,08 \mathrm{~ns}$ \\
\hline Absorción de agua (\%) & $4,66 \pm 0,00 \mathrm{~b}$ & $4,82 \pm 0,13 \mathrm{a}$ & $4,33 \pm 0,10 \mathrm{c}$ \\
\hline Hinchamiento (\%) & $0,70 \pm 0,00 \mathrm{~ns}$ & $0,74 \pm 0,04 \mathrm{~ns}$ & $0,66 \pm 0,03 \mathrm{~ns}$ \\
\hline Retrogradación (\%) & $41,36 \pm 0,19 \mathrm{a}$ & $70,71 \pm 0,21 b$ & $72,34 \pm 0,46 \mathrm{c}$ \\
\hline $\begin{array}{l}\text { Estabilidad al } \\
\text { descongelamiento (\%) }\end{array}$ & $4,10 \pm 1,18 \mathrm{c}$ & $1,32 \pm 0,32 \mathrm{a}$ & $1,29 \pm 0,71 b$ \\
\hline
\end{tabular}

La tabla 7 muestra las propiedades funcionales del almidón de las variedades de quinua, las muestras de almidón presentan una baja solubilidad de $5 \%$ y restringido poder de hinchamiento de $0,7 \%$ con $(\mathrm{p}>0,05)$ estadísticamente, comprobando que sus gránulos tienen fuerzas o enlaces de unión muy fuertes ${ }^{16}$; se mostró un alto rango de temperatura de gelatinización $\left(66-69^{\circ} \mathrm{C}\right)$ con $(\mathrm{p}>0,05)$ entre las variedades. La mayor temperatura de gelatinización es a $65^{\circ} \mathrm{C}$ y la menor a $55^{\circ} \mathrm{C}$; en cambio, el mayor tiempo de gelatinización es a 10 minutos y el menor a 5 minutos, o sea, el proceso de gelatinización guarda una relación directa entre temperatura, tiempo y variedad de quinua ${ }^{19}$. Además, presenta un ligero pico de viscosidad de 2006,50VC; $1521,50 \mathrm{VB}$ y un mínimo de $1009,00 \mathrm{cP}$ para VP. De la Rosa ${ }^{20}$ menciona que esta propiedad está relacionada con la distribución de cadenas de las moléculas de amilopectina. Por lo que desde el punto de vista de la viscosidad, la amilopectina con una mayor masa molar genera pastas más viscosas a altas temperaturas. Mientras que la amilosa es la responsable de altas viscosidades cuando la pasta se enfría. Teniendo como resultado una extrema estabilidad al descongelamiento de 4,10VC; 1,32VP; 1,29VB y una baja retrogradación de 41,36VC; 70,71VP y 72,34VB; para ambos una $(\mathrm{p}<0,05)$; sugiriendo su aplicación en productos sometidos a estos procesos. 


\section{Caracterización de los parámetros de calidad}

Tabla 8. Evaluación de anhidrido sulfuroso del almidón de quinua.

\begin{tabular}{|c|c|c|c|}
\hline & $\mathbf{V C}$ & VP & VB \\
\hline Anhidrido sulfuroso (ppm) & $11,52 \pm 1,81 \mathrm{~ns}$ & $12,16 \pm 0,91 \mathrm{~ns}$ & $12,80 \pm 0,00 \mathrm{~ns}$ \\
\hline Material no amiláceo (\%) & $2,00 \pm 0,00 \mathrm{~ns}$ & $1,00 \pm 0,00 \mathrm{~ns}$ & $1,00 \pm 0,00 \mathrm{~ns}$ \\
\hline \multicolumn{4}{|c|}{$\begin{array}{l}\text { *, ns: Indican significancia a } p<0,05 \text { y no significante. Valores con diferentes letras dentro de } \\
\text { cada columna denotan significancia en la prueba de Tu } \\
\text { key }(p<0,05) \text {, valores promedio de } 2 \text { repeticiones } \pm \text { desviación estándar. }\end{array}$} \\
\hline $\begin{array}{l}\text { LEYENDA PARA MATER } \\
\text { Material no amiláceo "A"= } \\
\text { Material no amiláceo "B" }= \\
\text { Material no amiláceo "C"= }\end{array}$ & $\begin{array}{l}\text { NO AMILÁCEO (ITI } \\
\text { edimento cubre un área nc } \\
\text { dimento cubre un área nc } \\
\text { dimento cubre un área no }\end{array}$ & $\begin{array}{l}\mathbf{2 0 9 . 0 7 0 )} \\
\text { or del } 15 \% \text { ) } \\
\text { or del } 30 \% \text { ) } \\
\text { or del } 50 \% \text { ) }\end{array}$ & \\
\hline
\end{tabular}

De la tabla 8 se puede observar que no existe diferencia significativa entre los porcentajes de anhidrido sulfuroso de las tres variedades de almidón de quinua. ITINTEC ${ }^{12}$ indica que para almidones no modificados, deberá cumplir un límite máximo de anhidrido sulfuroso a $60 \mathrm{ppm}$; los resultados obtenidos cumplen con lo indicado en la norma. Para interpretar los resultados de material no amiláceo citamos la Norma ITINTEC ${ }^{13}$ para almidones y féculas; el material no amiláceo son las impurezas o residuos que no tienen la estructura amilácea de los almidones que se detectan al sedimentar; el valor óptimo debe ser 1 y sólo lo obtienen la variedad Pasankalla y Junín, mientras que la variedad Collana obtiene 2. Entonces esta última contiene mayores impurezas y residuos.

\section{CONCLUSIONES}

- Se obtuvo y determinó el rendimiento de almidón de quinua a nivel de laboratorio, encontrándose que la variedad blanca tiene rendimiento de 30;64 \%, seguido por la Pasankalla roja con $26,82 \%$ y la Collana negra con $18,90 \%$. Existen diferencias significativas entre muestras y también entre especies si comparamos con los tubérculos, estos valores son considerados altos y muy rentables.

- Se caracterizó las propiedades funcionales del almidón extraído de tres variedades de quinua; con respecto a la viscosidad la variedad negra Collana genera pastas más viscosas; al evaluar Índice de solubilidad no se encontró diferencias.

- La evaluación de Índice de absorción de agua a las muestras, presentan valores mínimos, debido al reducido tamaño de partícula característico del almidón de quinua. El almidón de la variedad Collana es menos estable comparada a almidón de la variedad Junín y Pasankalla que tienen mayor estabilidad al procesos de descongelación.

- La información obtenida es importante para el procesamiento de las tres variedades de quinua, ya sea para la preparación de productos u otro tipo de uso, así como para predecir las características funcionales o nutricionales de los productos durante su almacenamiento. 


\section{AGRADECIMIENTO}

Los autores expresamos nuestro agradecimiento al Instituto de Investigación de la Facultad de Ingeniería en Industrias Alimentarias y Facultad de Ciencias Aplicadas de la Universidad Nacional del Centro.

\section{BIBLIOGRAFÍA}

1. Ruales, J. \& B. Nair. "Quinoa (Chenopodium quinoa Willd) an important Andean food crop. Arch". Latinoamer. Nutr, 1992; 42:232 - 241.

2. Ministerio de Agricultura. Variedades de quinua. Perú. 2013.

3. Matos, A., y Sánchez, F. "Determinación del rendimiento de almidón a partir en tres accesiones de quinua (Chenopodium quinoa Willd), Pasankalla, Salcedo INIA y Kancolla”. Universidad Peruana Unión, Tercer encuentro de Investigación Universitaria, Lima-Perú, 2011.

4. Ahamed, N.,Singhal, R., Kulkarni, P.y Pal, M. "Physicochemical and functional properties of Chenopodium quinoa starch". Botanical Reseurch Institute, Lucknow. India, 1996.

5. Repo-Carrasco, R.; Espinoza, C.; Jacobsen, S. E. "Nutritional value and use of the Andean crops quinoa (Chenopodium quinoa) and kañiwa (Chenopodium pallidicaule)". In: Food Review International, 2003.

6. Rojas, W., M. Pinto, JL. Soto y E. “Granos Andinos: Avances, logros y experiencias desarrolladas en quinua, cañahua y amaranto en Bolivia” Biodiversity International, Roma-Italia, 2010.

7. Official Methods of analysis of the Association of official Analytic Chemical of Official Analytical Chemists. USA. Editorial Boord. 2000.

8. Grace, M. "Elaboración de la yuca". Método para determinar temperatura de gelatinización. Organización de las Naciones Unidas para la Agricultura y la Alimentación (FAO). 1977, Roma. 116 pp.

9. Anderson, R., Conway, H.F., Pheiser, V.F. y Griffin, E.L. "Gelatinisation of corn grits by roll and extrusion cooking". Método para determinar poder de hinchamiento, índice de absorción de agua e índice de solubilidad. Cereal Science Today, 1969; 14: 4-1.

10. Lovedeep, K., Narpinder, S., Navdeep, S. "Some properties of potatoes y their starches". Método para determinar retrogradación. II Morphological, thermal y rheological properties of starches. Food Chemistry, 2002; 79: 183 - 192.

11. Sánchez, H., Solorza, J., Mendez, M. G., Paredes, L. O, Bello, P. L. "Isolation and partial characterization of Okenia (Okenia hypogaea) starch". Método para determinar estabilidad al descongelamiento. Starch, 2002; 54: 193-197.

12. ITINTEC. "Almidones y féculas". Método para determinar anhidrido sulfuroso. NTN 209.068. Instituto de Investigación Tecnológica Industrial y de Normas Técnicas (ITINTEC). Perú, 1997.

13. ITINTEC. "Almidones y féculas". Método para determinar material no amiláceo. NTN 209.070. Instituto de Investigación Tecnológica Industrial y de Normas Técnicas (ITINTEC). Perú, 1997. 
14. Singh, S., Singh, J., Kaur, L., Sodhi, S. y Gill, S. "Morphological, thermal and rheological properties of starches from different botanical sources A review". Food Chemistry, 2003; 81: 219-231.

15. Guizar, A. "Obtención y caracterización física y química del almidón de camote de cerro". Tesis para obtener maestría en ciencias de producción agrícola sustentable. Instituto Politécnico Nacional de Michoacán. 2009, México.70pp.

16. Raygada, M. "Caracterización del almidón de dos variedades de quinua (Chenopodium quinoa Willd): Kancolla y Chullpi”. Tesis para obtener el grado de Magister Scientiae. Universidad Nacional Agraria La Molina. 2001, Lima-Perú. 93pp.

17. ISI. "Determination of starch size distribution by screening". ISI 32-1e. In: Laboratory methods. Science Park, Aarhus, Dinamarca, International Starch Institute (ISI), 2000.

18. ICONTEC. "Alimentos para animales". Determinación del contenido de fósforo. Método espectrofotométrico. Instituto Colombiano de Normas Técnicas y Certificación (ICONTEC). 2001, Bogotá. NTC 4981.

19. Ortega, M. "Propiedades viscoelásticas y reológicas estacionarias de suspensiones de almidón nativo de quinua". Tesis para optar al grado de magister en ciencia de los alimentos. Facultad de Ciencias Químicas y Farmacéuticas de la Universidad de Chile. 2008, Chile. 39pp.

20. De la Rosa. J. "Análisis fisicoquímico, estructural y molecular de almidones de diferentes variedades de maíz azul". Tesis para obtener el grado de maestría en ciencias en desarrollo de productos bióticos. 2009, México. 86pp. 